\title{
Reticular chemistry studies on CuCN derivatives for new luminescent materials
}

Emanuele Priola $^{1}$, Diana Eliano ${ }^{1}$, Michele Chierotti ${ }^{1}$, Elisabetta Bonometti ${ }^{1}$, Alessia Giordana ${ }^{1}$, Francesca Grifasi ${ }^{1}$

${ }^{1}$ Department Of Chemistry, University Of Turin, Turin, Italy

E-mail: emanuele.priola@unito.it

The construction of coordination polymers has been rationalized through the use of database and a topological approach. The reticular chemistry is based on the specific geometry of tectons that, by combination, form a predictable topology. This abstract procedure, in presence of nodes with a specific coordination form and of directional interactions, allows to obtaining a desired topology. This is often connected with the properties of the materials, like porosity, NLO properties and magnetism. Cyanide is a good ligand in reticular chemistry: its linearity in coordination bond makes equivalent both the coordination form and the coordination polyhedron of metal centers, and permits to clarify the derived structures. Although the lot of work done in recent years [1], some systems are very complex and less predictable than others. For example, the $\mathrm{Cu}(\mathrm{I}) \mathrm{CN}$ system is interesting because this salt, that forms linear polymer in the solid state [2], became very complex in presence of ancillary ligands. $\mathrm{Cu}(\mathrm{I})$ metal centers can have different coordination numbers and different coordination geometries. On the other hand, the cyanide itself has a multiform behavior in this system: the $\mu 2$ bridging mode, the terminal one and the combination of different bridging behaviors have been observed. For this reasons, a clearly predictable nodal geometry and a defined topological derivation is difficult to obtain, and a lot of work has still to be done. We focalized our attention on three different aspects. The first one is the presence of a terminal or briging ligand with different sterical requirements: to obtain some clarifying informations, we synthesized seven new derivatives of substituted and unsubstituted thioureas, and from their characterization and from a statistical analysis of Databases we correlate the nature of the ligand and the topology of the derived compounds. The second aspect is the effect of the partial substitution of Cu(I) with $\mathrm{Cu}(\mathrm{II})$ ion on the topology: to pursue a rationalization, we synthesized and structurally characterized six mixed $\mathrm{Cu}(\mathrm{I}) / \mathrm{Cu}(\mathrm{II})$ coordination polymers that will be commented. The third aspect is the presence of bi-chelating ligands of different rigidity and symmetry (2,2'-terpyridine and 2-(2'- pyridyl)-1,8-naphthyridine) on the topology of the derived coordination polymers. This family of ligands, differently from the monochelating ones that follow the usual $1 \mathrm{D}$ wavy topology typical of terminal ligands, allows to construct interesting 2D-nets with $\{63\}$ point symbol [3] and the presence of cuphrophilic interaction based on Secondary Building Units. We synthesized and structurally characterized two new compounds and the effects that favour the previous cited topology will be commented. All the previous analysis have been undertaken in the search of optimal luminescence for LED materials, and a full solid state luminescence characterization of all the $16 \mathrm{CuCN}$ derivatives here presented has been done. In this communication, we will comment the principal effects influencing this property.

[1] Alexandrov E.V., Virovets A.V., Blatov V.A., Peresypkina E.V. (2015) Chem. Rev. 115, 12286-12319.

[2] Bayse C. A., Brewster T.P., Pike R.D. (2009) Inorg. Chem. 48, 174-182.

[3] Blatov V.A., O’Keeffe M., Proserpio D. M. (2010) CrystEngComm 12, 44-48.

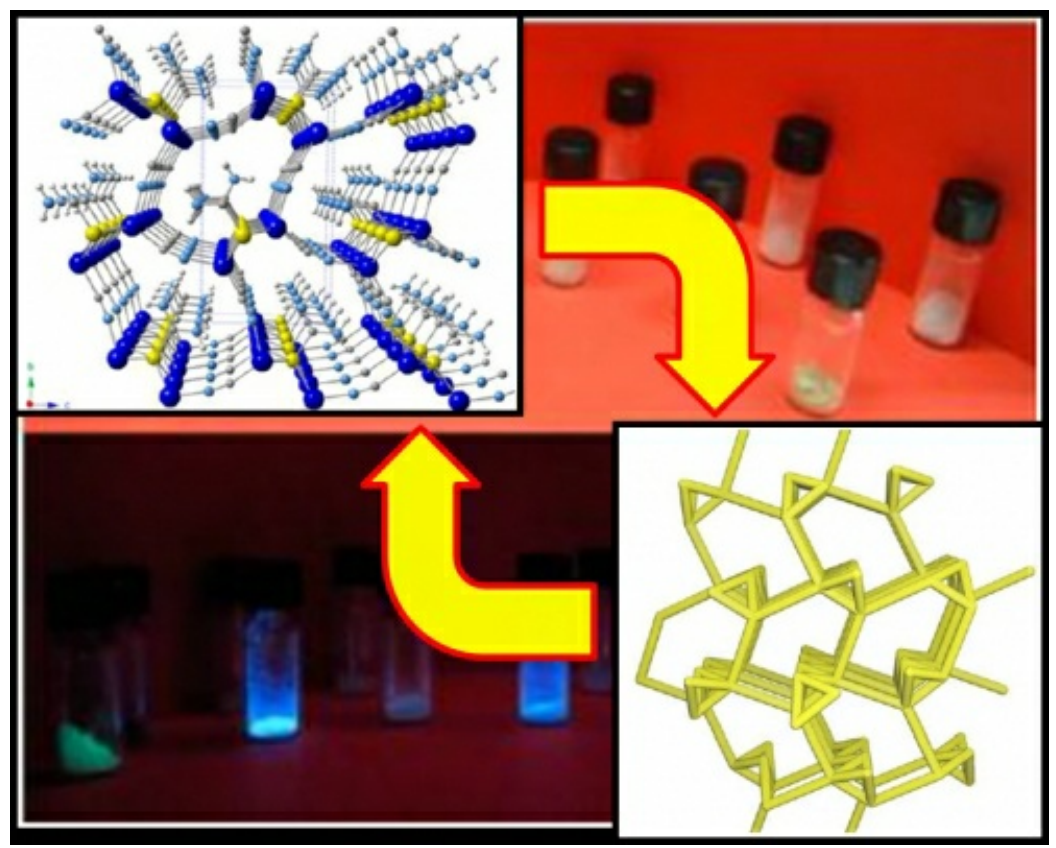

Keywords: reticular chemistry. coordination polymers, luminescence 